\title{
Discovery of 1323 s pulsations from RX J0103.6-7201: The longest period X-ray pulsar in the SMC ${ }^{\star}$
}

\author{
F. Haberl and W. Pietsch
}

\author{
Max-Planck-Institut für extraterrestrische Physik, Giessenbachstraße, 85748 Garching, Germany \\ e-mail: fwh@mpe.mpg.de
}

Received 2 December 2004 / Accepted 16 March 2005

\begin{abstract}
XMM-Newton archival observations of the Be/X-ray binary candidate RXJ0103.6-7201 revealed pulsations with a period of $\sim 1323$ s. This makes RX J0103.6-7201 the X-ray pulsar with the longest period known in the Small Magellanic Cloud (SMC). More than 150 X-ray observations of RX J0103.6-7201 by ROSAT, Chandra and XMM-Newton show flux variations by a factor of 50 on time scales of days to years. Using the accurate positions obtained from ACIS-I images, the optical counterpart is identified as a $V=14.6$ mag emission line star. EPIC spectra of RX J0103.6-7201 above $1 \mathrm{keV}$ are consistent with an absorbed power-law with column density between $(6-9) \times 10^{21} \mathrm{~cm}^{-2}$, except during one observation when an extraordinary high value of $1.1 \times 10^{23} \mathrm{~cm}^{-2}$ was measured which strongly attenuated the power-law emission below $3 \mathrm{keV}$. A soft excess between 0.5 and $1.0 \mathrm{keV}$ is evident in the spectra which becomes best visible in the highly absorbed spectrum. The soft component can be reproduced by a thermal plasma emission model with its luminosity strongly correlated with the total intrinsic source luminosity. Including results from three other SMC Be/X-ray binaries extends the linear correlation over three orders of magnitude in source intensity, strongly suggesting that the same mechanism is responsible for the generation of the soft emission.
\end{abstract}

Key words. galaxies: individual: small Magellanic Cloud - stars: neutron - X-rays: binaries - X-rays: galaxies X-rays: individual: RX J0103.6-7201- stars: emission-line, Be

\section{Introduction}

High mass X-ray binaries (HMXBs) are binary systems consisting of a compact stellar object accreting matter from a high mass early type star. In most cases the detection of pulsations in the X-ray flux with periods in the range of $0.07 \mathrm{~s}$ to $1500 \mathrm{~s}$ reveals a spinning neutron star orbiting an OB supergiant or a Be star. The large population of HMXBs makes the SMC unique among the galaxies of the Local Group. First, the absolute number of known HMXBs is similar to that in the Milky Way, despite the comparatively small mass of the SMC and second, while the ratio of supergiant to Be binaries in the Milky Way is $1 / 3.5$ as found from a detailed literature search, the vast majority of HMXBs in the SMC has a Be star counterpart.

Currently 48 X-ray pulsars are known in the SMC (Coe et al. 2005), from which 45 are most likely in HMXB systems as suggested by their X-ray properties (Haberl \& Pietsch 2004). Optical identifications confirmed 33 Be counterparts while only one supergiant system (SMCX-1) is known. Additional candidates for Be/X-ray binaries were found by Haberl \& Sasaki (2000) from correlations of ROSAT catalogues

* Based on observations with XMM-Newton, an ESA Science Mission with instruments and contributions directly funded by ESA Member states and the USA (NASA). of SMC sources with lists of $\mathrm{H} \alpha$ emission line objects (Meyssonnier \& Azzopardi 1993, hereafter MA93) and by Sasaki et al. (2003).

One of the Be/X-ray binary candidates is RX J0103.6-7201, its ROSAT position (with an uncertainty of $4^{\prime \prime}$ ) is consistent with the emission line object [MA93] 1393 (Haberl \& Sasaki 2000). This hard ROSAT source was detected in PSPC (Haberl et al. 2000) and HRI (Sasaki et al. 2000) pointed observations of the SMC. From three ROSAT observations a factor of three flux variability was seen on time scales of years. A first analysis of early XMM-Newton data was performed by Sasaki et al. (2003). RX J0103.6-7201 is located close to the supernova remnant 1E0102-72.3 in the North-East of the SMC which is frequently observed as calibration source for the instruments on-board XMM-Newton and Chandra. About 150 Chandra observations were performed with RX J0103.6-7201 located on various ACIS CCD chips which are readily available from the Chandra archive. In addition twelve XMM-Newton observations with the EPIC instruments in various readout modes makes RX J0103.6-7201 the most frequently observed extragalactic X-ray binary. We analyzed the temporal and spectral properties of RX J0103.6-7201 and present the results in this paper. 


\section{Data analysis}

The Chandra ACIS calibration observations of 1E0102-72.3 are typically of $8 \mathrm{ks}$ exposure (five observations have $20 \mathrm{ks}$ ) and were used to determine an accurate position (Sect. 2.4) and to investigate the flux history of RX J0103.6-7201 (Sect. 2.3). The twelve XMM-Newton EPIC observations performed until 2004, Oct. 15 have longer exposures of 20-30 ks (only one exception with $13 \mathrm{ks}$ ). The EPIC-pn (Strüder et al. 2001) and EPIC-MOS (Turner et al. 2001) data were, therefore, used for a spectral and temporal analysis, in particular to search for X-ray pulsations. During five of the EPIC observations (here as observation a single pointing within a satellite revolution is defined) two different CCD readout modes were used for the pn camera (switching between FF: full frame, LW: large window and SW: small window mode) while the MOS cameras were operated in LW mode during the whole observation (only during revolution 433 and 447 part of the time was spent in timing mode). Different optical light blocking filters (thin, medium and thick) were chosen in different revolutions (see also Table 1). During revolutions 616 and 803 the MOS LW mode field of view did not cover RX J0103.6-7201. Also the MOS timing mode observations cannot be used. The data were processed using the XMM-Newton analysis package SAS 6.0.0 to produce the photon event files and binned data products such as images and spectra. Events for spectral and temporal analysis of RX J0103.6-7201 were extracted from circular regions (radius $20^{\prime \prime}$ ) around the source position and from nearby sourcefree regions for background spectra. Photon arrival times were corrected to the solar system barycentre. For the purpose of searching for relatively long periods of seconds to tens of minutes as observed from Be/X-ray binaries the EPIC-pn data from one satellite revolution with different readout modes were merged. MOS and pn data were combined for the common time intervals, i.e. MOS data at the beginning of an observation when the pn camera was still calculating the offset map (and also when the readout mode was changed) were disregarded.

\subsection{X-ray pulsations}

We performed a timing analysis of the EPIC data of RX J0103.6-7201, searching for pulsations in the broad energy band $(0.3-8.0 \mathrm{keV})$ using the Rayleigh $Z_{1}^{2}$ technique in the range $1-3000 \mathrm{~s}$. Strong peaks in the probability density function were found from the observations in revolutions 521 (pn SW+LW and MOS1/2 LW), 616 (pn SW+LW), 721 (pn FF and MOS1/2 LW) and 803 (pn LW) with $Z_{1}^{2}$ of 93.2, 77.9, 68.4 and 29.9, respectively. The maximum $Z_{1}^{2}$ of 93.2 derived for revolution 521 corresponds to a period detection confidence of $1.0-1.3 \times 10^{-16}$ (disregarding the detection in other observations). Figure 1 shows two example periodograms (top) together with the EPIC X-ray light curves folded with the most likely period value derived from the $Z^{2}$ test. To investigate energy dependencies of the pulse profile, light curves in the $0.3-1.5 \mathrm{keV}$ (soft), $1.5-8.0 \mathrm{keV}$ (hard) and the total $0.3-8.0 \mathrm{keV}$ energy bands were created. Hardness ratios derived by dividing the pulse profiles in the hard and soft bands show significant variations caused by weaker pulse modulations in the soft band compared to the hard band. This is particularly evident during the revolution 616 observation where no significant modulation was seen in the soft band. Period errors $(1 \sigma)$ were determined by $\chi^{2}$ fitting of a sine wave to the total light curves with $80 \mathrm{~s}$ binning, taking into account long term trends visible in the light curves by adding polynomial components to the sine model light curve. We also tested the inclusion of a second harmonic component as might be suggested by the analysis shown in Fig. 1. This did not improve the results because the major uncertainties in the determination of the long pulse period arise from the superposed long term trends. The pulse period history for the four observations is shown in Fig. 2. No significant change in the pulse period is visible consistent with a constant period of $(1323.2 \pm 5.8) \mathrm{s}$. However, the large errors are still compatible with large spin-up or spin-down with up to $\pm 13 \mathrm{~s}_{\text {year }}{ }^{-1}$.

\subsection{X-ray spectra}

Pulse phase averaged EPIC-pn and -MOS spectra were extracted using pattern $0-4$ (single+double pixel) and pattern 0-12 (single - quadruple) events, respectively, and binned to obtain at least 50 counts per bin. For observations split into parts with different readout modes (with different spectral response) separate spectra were obtained. Nine EPIC observations yielded a sufficiently high number of counts for a spectral analysis. All spectra for each observation were fit simultaneously (using XSPEC v11.3) with the same model only allowing a free normalization factor between the different instruments and readout modes (which cover different time intervals with varying source intensity). Errors were determined for $90 \%$ confidence levels. A simple power-law model including photoelectric absorption by matter with solar abundance reproduces the data well above $1 \mathrm{keV}$ while at lower energies a soft excess is apparent, in particular in the EPIC-pn spectra. This soft component is clearly visible during the XMM-Newton observation of revolution 888 when the power-law component was highly absorbed and did not contribute below $3 \mathrm{keV}$. The EPIC spectra of this observation were used to investigate the nature of the soft component.

To fit the soft component, a black-body model and a model for a hot plasma in collisional equilibrium (Mewe et al. 1985, MEKAL model in XSPEC) were compared. These were used in the past to reproduce low energy excesses seen in spectra of SMC Be/X-ray binaries (Sasaki et al. 2003; Haberl et al. 2003; Majid et al. 2004). The EPIC spectra of RX J0103.6-7201 from revolution 888 were fit with these models between 0.2 and $2.0 \mathrm{keV}$ where the power-law contribution can be neglected. This results in $\chi^{2} /$ d.o.f. values of $19.04 / 15$ and $14.50 / 12$ for the black-body and the thermal model, respectively. The thermal plasma model better reproduces the structures in the spectrum which are probably caused by emission lines around $650 \mathrm{eV}$ and $850 \mathrm{eV}$. Therefore, the thermal model was used as additional component to the power-law to fit the full EPIC spectra from all observations.

For the revolution 888 spectra two individual column density values were used for the two spectral components resulting 

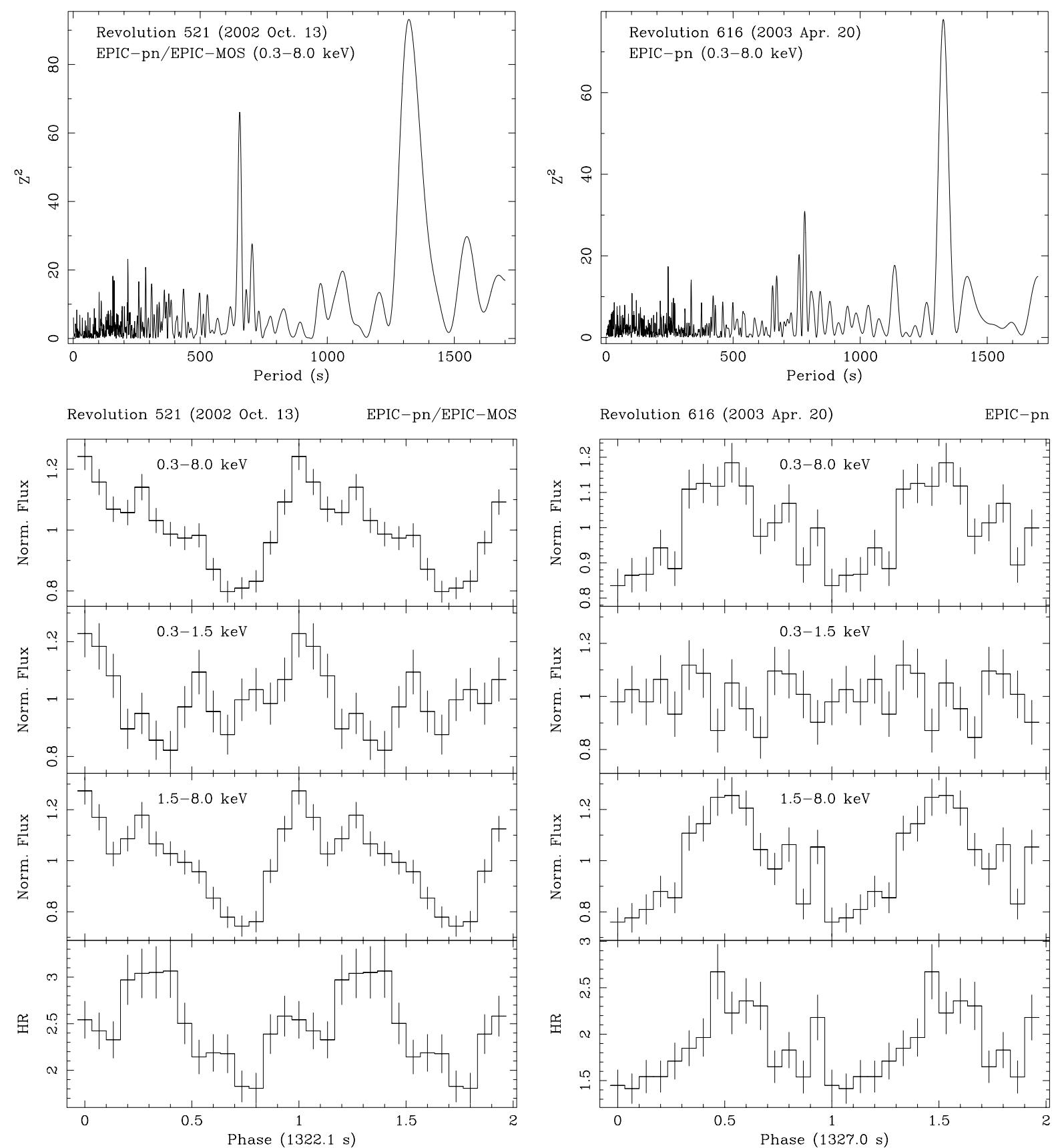

Fig. 1. Periodograms of the $Z_{1}^{2}$ test from two XMM-Newton observations of RX J0103.6-7201 (top). Corresponding pulse profiles folded with the most likely period for different energy bands, together with hardness ratios (HR) are shown in the bottom panels. For sinusoidal variations pulsed fractions in the $0.3-8.0 \mathrm{keV}$ band are $15.7 \pm 1.7 \%$ (rev. 521) and $13.2 \pm 2.2 \%$ (rev. 616).

in very different best fit values for $N_{\mathrm{H}}$ of $\left(5.7_{-2.3}^{+1.9}\right) \times 10^{21} \mathrm{~cm}^{-2}$ and $107 \pm 28 \times 10^{21} \mathrm{~cm}^{-2}$ for the thermal and the power-law component, respectively. For all other observations the $N_{\mathrm{H}}$ values were not significantly different and therefore the same (but free to vary in the fit) value was used for both components. Also the temperature was fixed at the value derived from the revolution 888 observation $(k T=0.15 \pm 0.04 \mathrm{keV})$. In Table 1 the results for the spectral fits are summarized: photon in$\operatorname{dex} \gamma$, absorption column density $N_{\mathrm{H}}$, emission measure $E M=$ $\int n_{\mathrm{H}} n_{\mathrm{e}} \mathrm{d} V$, together with fluxes and source luminosities and reduced $\chi^{2}$ values per degree of freedom (d.o.f.). In Fig. 3 the EPIC spectra together with the best fit models are plotted.

\subsection{Energy dependence of pulsations}

The pulsed fraction observed from RX J0103.6-7201 during revolution 616 is largely reduced (if present at all) in the $0.3-1.5 \mathrm{keV}$ band compared to the $1.5-8.0 \mathrm{keV}$ band. However, the observation from revolution 521 shows that there can be still a large contribution from the power-law component in the soft band. The observation from revolution 888 where the soft component was completely isolated is ideally suited to investigate the pulse profiles of both spectral components separately. This is shown in Fig. 4 and although the statistics is relatively 


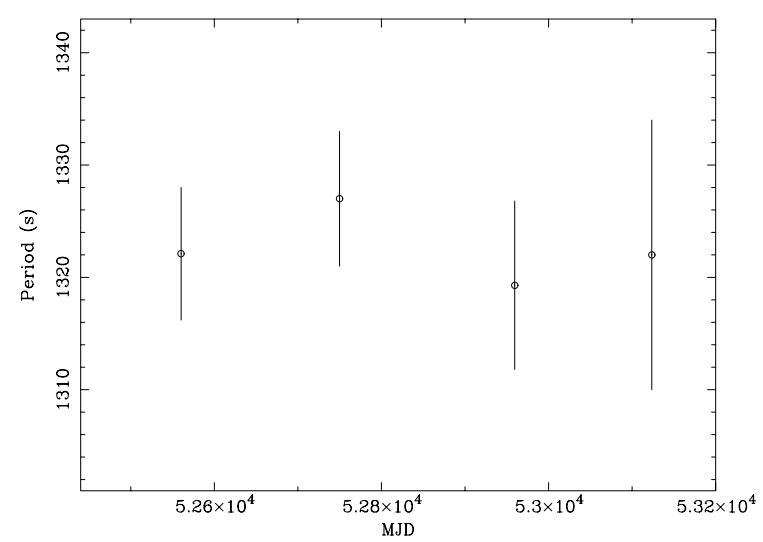

Fig. 2. Pulse period history of RX J0103.6-7201 over 1.5 years. The pulse periods of $1322.1 \pm 5.9,1327.0 \pm 6.0,1319.3 \pm 7.5$ and $1322 \pm$ 12 were derived from the EPIC data from revolutions 521, 616, 721 and 803 , respectively.

poor, pulsations can be detected above $1.5 \mathrm{keV}$ while below they are strongly reduced or may not be present at all.

\subsection{Long-term intensity variations}

To look at intensity variations of RX J0103.6-7201 on time scales of days to years we converted ROSAT and Chandra count rates obtained from source detection analyses into 0.2-10.0 keV fluxes. We assumed a power-law plus MEKAL spectrum with photon index 0.9 , column density of $6.5 \times$ $10^{21} \mathrm{~cm}^{-2}$ and temperature $0.15 \mathrm{keV}$, typical values as derived from the EPIC spectra. Similarly, for EPIC observations with insufficient statistical quality (short exposure and/or low source flux) count-rates were used. For the estimates of ROSAT fluxes relatively large systematic errors may be introduced due to the strong influence of the assumed column density on the soft $0.1-2.4 \mathrm{keV}$ band count rate to luminosity conversion (an additional $N_{\mathrm{H}}$ of $1.0 \times 10^{21} \mathrm{~cm}^{-2}$ changes the conversion factor by $12 \%$ ). The measured fluxes as function of time over nearly 13 years are plotted in Fig. 5. There seems to be a base flux level of $\sim 7.6 \times 10^{-13} \mathrm{erg} \mathrm{cm}^{-2} \mathrm{~s}^{-1}$ below which the source was never seen. The maximum flux of $3.7 \times$ $10^{-12} \mathrm{erg} \mathrm{cm}^{-2} \mathrm{~s}^{-1}$ is a factor of 50 higher and was reached during the XMM-Newton observation of revolution 247 (second part with EPIC-pn in FF mode). A similar outburst level was seen also during the third ROSAT observation, four Chandra and one other XMM-Newton observation. Chandra observations were often performed in blocks spread over $\sim 0.7$ days during which the source stayed at a similar intensity level, indicating that high and low states last longer than that. The source was generally brighter (both, low and high intensity levels decreased) during the first half as compared to the second half of the last five years (Fig. 5) which may be related to long-term changes in the properties of the circum-stellar matter around the mass donor star.

\subsection{Source identification}

X-ray source positions were obtained from the analysis of all observations where the source was detected in the ACIS-I CCDs. The average position from 71 observations with a standard deviation of $11^{\prime \prime} 1$ is only 0.'07 away from the 2MASS object $01033752-7201330$ at $\mathrm{RA}=01^{\mathrm{h}} 03^{\mathrm{m}} 37.524$ and Dec $=$ $-72^{\circ} 01^{\prime} 33^{\prime \prime}$.03 (J2000.0) for which an error of 0.'11 is given (Cutri et al. 2003). For comparison the average EPIC position is within $0 . ' 8$ of the 2MASS position. This star is covered by the SMC UBVR CCD survey of Massey (2002) and also listed in the $U B V I$ photometry catalogue of Zaritsky et al. (2002). Optical positions and available magnitudes are summarized in Table 2. The star can be identified with the emission line object ([MA93] 1393, Meyssonnier \& Azzopardi 1993). The presence of a 14.6 Vmag star inside the error circle of the Chandra position clearly establishes it as the optical counterpart of the X-ray source.

The inferred effective temperature and bolometric luminosity of the optical counterpart given by Massey (2002) suggest a spectral type of $05 \mathrm{~V}$. Assuming this spectral type the measured $B-V$ index imply $E(B-V)$ of 0.32 and $N_{\mathrm{H}}=1.9 \times$ $10^{21} \mathrm{~cm}^{-2}$.

\section{Discussion}

\section{1. $R X$ J0103.6-7201, a Be/X-ray binary pulsar}

The discovery of a 1323 s modulation in the X-ray flux from RX J0103.6-7201 increases the number of pulsars in the SMC to 49 from which up to 46 are most likely neutron stars in HMXB systems. The pulse period of $1323 \mathrm{~s}$ is the longest known from SMC pulsars. It considerably exceeds that of AX J0049.4-7323 (=RX J0049.7-7323) (756 s; Yokogawa et al. 2003) and is similar to that of RX J0146.9+6121 (Haberl et al. 1998) in the Milky Way. Optical spectra of 33 counterparts from HMXBs in the SMC identify the majority as Be-star binaries (Coe et al. 2005), while only one (SMC X-1) is a supergiant system. Brightness and colours of the optical counterpart and the presence of $\mathrm{H} \alpha$ emission (MA93) strongly suggest that also RX J0103.6-7201 is a Be/X-ray binary system.

The X-ray spectrum of RXJ0103.6-7201 is typical for $\mathrm{Be} / \mathrm{X}$-ray binaries in the SMC. The power-law photon index of $\sim 0.9$ is well within the relatively narrow range observed from these systems (Haberl \& Pietsch 2004). The absorbing column density was found to be similar during most observations yielding $(6-9) \times 10^{21} \mathrm{~cm}^{-2}$, except during the XMM-Newton observation in revolution 888 when $N_{\mathrm{H}}$ exceeded $10^{23} \mathrm{~cm}^{-2}$. Such high values are usually seen only from supergiant HMXBs when the X-ray source is viewed through the dense innermost parts of the stellar wind of the supergiant before or after eclipse (e.g. 4U1700-37 and Vela X-1; Haberl et al. 1989; Sako et al. 1999). This suggests that we may view the system of RX J0103.6-7201 at high inclination and during 2004, Oct. 14 (revolution 888) the line of sight to the X-ray source crossed the wind region very close to the Be star. Since $\mathrm{Be} / \mathrm{X}$-ray binaries are much wider systems in comparison to supergiant systems such cases should be very rare. Indeed, 
Table 1. XMM-Newton EPIC spectral fit results.

\begin{tabular}{|c|c|c|c|c|c|c|c|c|}
\hline $\begin{array}{l}\text { Instr. mode } \\
\text { filter }\end{array}$ & $\begin{array}{l}\operatorname{Rate}^{(1)} \\
\operatorname{cts~s}^{-1}\end{array}$ & $\begin{array}{c}N_{\mathrm{H}} \\
10^{21} \mathrm{~cm}^{-2}\end{array}$ & $\gamma$ & $\begin{array}{c}E M \\
10^{58} \mathrm{~cm}^{-3}\end{array}$ & $\begin{array}{c}\operatorname{Flux}^{(1)} \\
\operatorname{erg~cm}^{-2} \mathrm{~s}^{-1}\end{array}$ & $\begin{array}{c}L_{\mathrm{x}}^{(2)} \\
\mathrm{erg} \mathrm{s}^{-1}\end{array}$ & $\begin{array}{c}L_{\mathrm{x}}^{\mathrm{i}(3)} \\
\mathrm{erg} \mathrm{s}^{-1}\end{array}$ & $\chi_{\mathrm{r}}^{2} /$ d.o.f. \\
\hline \multicolumn{9}{|c|}{2000 Apr. 16/17 (Revolution 065) } \\
\hline PN FF thin & 0.177 & $6.5 \pm 1.5$ & $0.88 \pm 0.07$ & 6.93 & $1.61 \times 10^{-12}$ & $6.87 \times 10^{35}$ & $2.00 \times 10^{36}$ & \\
\hline M1 LW thin & 0.052 & & & 7.18 & $1.66 \times 10^{-12}$ & $7.13 \times 10^{35}$ & $2.08 \times 10^{36}$ & \\
\hline M2 LW thin & 0.051 & & & 6.90 & $1.60 \times 10^{-12}$ & $6.84 \times 10^{35}$ & $2.00 \times 10^{36}$ & \\
\hline PN FF medium & 0.156 & & & 6.10 & $1.41 \times 10^{-12}$ & $6.04 \times 10^{35}$ & $1.76 \times 10^{36}$ & \\
\hline M1 LW medium & 0.048 & & & 6.56 & $1.52 \times 10^{-12}$ & $6.50 \times 10^{35}$ & $1.90 \times 10^{36}$ & \\
\hline M2 LW medium & 0.049 & & & 6.63 & $1.54 \times 10^{-12}$ & $6.57 \times 10^{35}$ & $1.92 \times 10^{36}$ & $1.29 / 209$ \\
\hline \multicolumn{9}{|c|}{2001 Apr. 14/15 (247) } \\
\hline PN SW thin & 0.413 & $6.2 \pm 0.4$ & $0.84 \pm 0.03$ & 22.1 & $3.59 \times 10^{-12}$ & $1.54 \times 10^{36}$ & $5.61 \times 10^{36}$ & \\
\hline PN FF thin & 0.376 & & & 20.0 & $3.25 \times 10^{-12}$ & $1.39 \times 10^{36}$ & $5.08 \times 10^{36}$ & \\
\hline M1 LW thin & 0.121 & & & 21.9 & $3.55 \times 10^{-12}$ & $1.52 \times 10^{36}$ & $5.55 \times 10^{36}$ & \\
\hline M2 LW thin & 0.126 & & & 21.9 & $3.57 \times 10^{-12}$ & $1.53 \times 10^{36}$ & $5.58 \times 10^{36}$ & $1.24 / 347$ \\
\hline \multicolumn{9}{|c|}{2001 Dec. $25 / 26$ (375) } \\
\hline PN SW thin & 0.189 & $7.0 \pm 1.6$ & $0.90 \pm 0.08$ & 6.03 & $1.77 \times 10^{-12}$ & $7.56 \times 10^{35}$ & $1.94 \times 10^{36}$ & \\
\hline M1 LW thin & 0.064 & & & 6.86 & $2.01 \times 10^{-12}$ & $8.61 \times 10^{35}$ & $2.20 \times 10^{36}$ & \\
\hline $\mathrm{M} 2 \mathrm{LW}$ thin & 0.063 & & & 6.73 & $1.97 \times 10^{-12}$ & $8.44 \times 10^{35}$ & $2.16 \times 10^{36}$ & $1.19 / 177$ \\
\hline \multicolumn{9}{|l|}{2002 Oct. 13 (521) } \\
\hline PN SW thin & 0.244 & $6.8 \pm 1.0$ & $0.85 \pm 0.05$ & 12.1 & $2.35 \times 10^{-12}$ & $1.01 \times 10^{36}$ & $3.27 \times 10^{36}$ & \\
\hline PN LW thin & 0.289 & & & 14.7 & $2.88 \times 10^{-12}$ & $1.23 \times 10^{36}$ & $4.00 \times 10^{36}$ & \\
\hline M1 LW thin & 0.096 & & & 15.4 & $3.00 \times 10^{-12}$ & $1.28 \times 10^{36}$ & $4.16 \times 10^{36}$ & \\
\hline M2 LW thin & 0.094 & & & 15.0 & $2.91 \times 10^{-12}$ & $1.24 \times 10^{36}$ & $4.04 \times 10^{36}$ & $1.08 / 185$ \\
\hline \multicolumn{9}{|c|}{2002 Dec. 14 (552) } \\
\hline PN SW thin & 0.244 & $6.5 \pm 1.3$ & $0.91 \pm 0.09$ & 4.83 & $5.50 \times 10^{-13}$ & $2.36 \times 10^{35}$ & $1.12 \times 10^{36}$ & \\
\hline PN LW thin & 0.289 & & & 5.74 & $6.56 \times 10^{-13}$ & $2.81 \times 10^{35}$ & $1.33 \times 10^{36}$ & \\
\hline M1 LW thin & 0.096 & & & 5.63 & $6.41 \times 10^{-13}$ & $2.75 \times 10^{35}$ & $1.30 \times 10^{36}$ & \\
\hline M2 LW thin & 0.094 & & & 5.83 & $6.64 \times 10^{-13}$ & $2.84 \times 10^{35}$ & $1.35 \times 10^{36}$ & $0.82 / 63$ \\
\hline \multicolumn{9}{|c|}{2003 Apr. 20/21 (616) } \\
\hline PN SW medium & 0.200 & $8.4 \pm 1.2$ & $0.96 \pm 0.06$ & 30.9 & $1.88 \times 10^{-12}$ & $8.04 \times 10^{35}$ & $6.40 \times 10^{36}$ & \\
\hline PN LW medium & 0.166 & & & 26.9 & $1.63 \times 10^{-12}$ & $6.98 \times 10^{35}$ & $5.55 \times 10^{36}$ & $0.99 / 111$ \\
\hline \multicolumn{9}{|c|}{2003 Nov. 16 (721) } \\
\hline PN FF thick & 0.143 & $6.2 \pm 1.1$ & $0.91 \pm 0.05$ & 8.53 & $1.32 \times 10^{-12}$ & $5.65 \times 10^{35}$ & $2.14 \times 10^{36}$ & \\
\hline M1 LW thin & 0.050 & & & 9.55 & $1.48 \times 10^{-12}$ & $6.32 \times 10^{35}$ & $2.40 \times 10^{36}$ & \\
\hline M2 LW thin ${ }^{(4)}$ & 0.036 & & & 7.66 & $1.18 \times 10^{-12}$ & $5.07 \times 10^{35}$ & $1.92 \times 10^{36}$ & $1.48 / 125$ \\
\hline \multicolumn{9}{|c|}{2004 Apr. 28 (803) } \\
\hline PN LW thick & 0.030 & $6.6 \pm 1.7$ & $1.12 \pm 0.15$ & 3.28 & $2.82 \times 10^{-13}$ & $1.21 \times 10^{35}$ & $7.21 \times 10^{35}$ & $0.72 / 25$ \\
\hline \multicolumn{9}{|c|}{2004 Oct. $14(888)^{(5)}$} \\
\hline PN LW thick & 0.055 & $107 \pm 28$ & $1.03 \pm 0.34$ & 4.19 & $7.31 \times 10^{-13}$ & $3.13 \times 10^{35}$ & $1.34 \times 10^{36}$ & \\
\hline M1 LW thick & 0.013 & & & 4.40 & $7.67 \times 10^{-13}$ & $3.28 \times 10^{35}$ & $1.40 \times 10^{36}$ & \\
\hline M2 LW thick ${ }^{(4)}$ & 0.008 & & & 3.18 & $5.56 \times 10^{-13}$ & $2.38 \times 10^{35}$ & $1.02 \times 10^{36}$ & $1.48 / 45$ \\
\hline
\end{tabular}

(1) Net count rates and observed total fluxes in the $0.2-10.0 \mathrm{keV}$ band.

(2) X-ray $0.2-10.0 \mathrm{keV}$ luminosity (including absorption) for a distance of $60 \mathrm{kpc}$.

(3) Source intrinsic X-ray luminosity in the $0.2-10.0 \mathrm{keV}$ band (corrected for absorption).

(4) Source on bad CCD column.

(5) Individual column density for the thermal plasma component $N_{\mathrm{H}}=\left(5.7_{-2.3}^{+1.9}\right) \times 10^{21} \mathrm{~cm}^{-2}$. The temperature of the thermal plasma model component was determined from this observation to $k T=0.15 \pm 0.04 \mathrm{keV}$ and fixed in the fits to the other observations.

RX J0103.6-7201 is to our knowledge the only Be/X-ray binary which has shown a spectrum with such extreme absorption. Alternatively absorption by dense material close to the neutron star cannot be ruled out, but may be expected to occur more often.

\subsection{Soft emission in the $X$-ray spectra of Be/X-ray binaries}

A soft spectral component in the X-ray spectrum of RX J0103.6-7201 was first reported by Sasaki et al. (2003) 

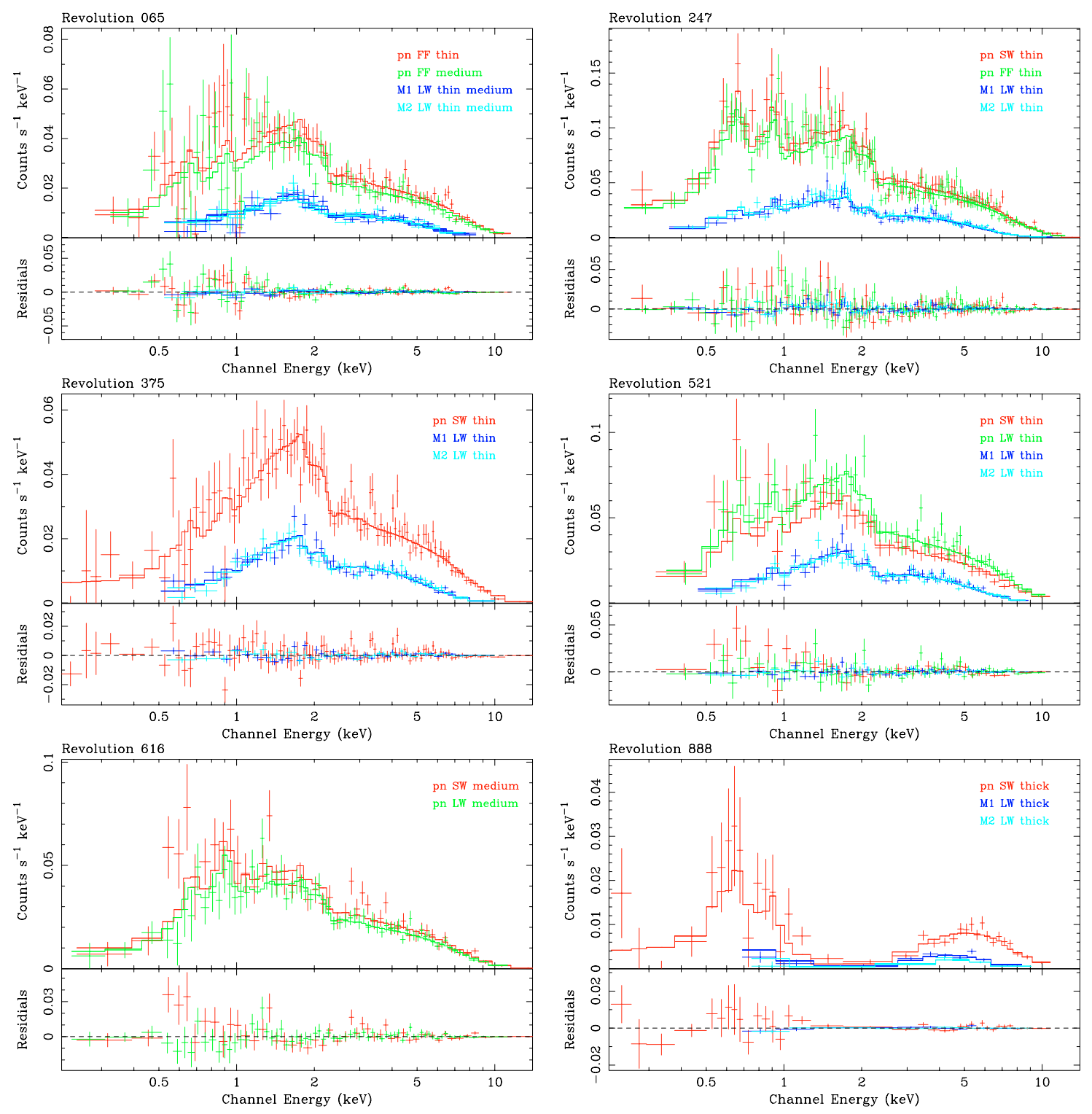

Fig. 3. EPIC spectra of RX J0103.6-7201 from six observations.

who analyzed the XMM-Newton data from revolution 247 and fit the EPIC-pn spectrum with thermal plasma and powerlaw components. This soft emission became clearly visible in the revolution 888 observation when both spectral components were separated into different energy bands. From Table 1 it is evident that the intensity of the soft component, given as emission measure (which directly reflects the intrinsic luminosity of the soft component) in the range of (3-30) $\times 10^{58} \mathrm{~cm}^{-3}$ strongly correlates with the intrinsic source luminosity. This is demonstrated in the top panel of Fig. 6 where the luminosity of the soft component is plotted versus the total source luminosity.

Similar soft emission was also observed from the pulsars EXO 053109-6609.2 with XMM-Newton (Haberl et al. 2003) and RX J0059.2-7138 in a simultaneous ROSAT/ASCA observation (Kohno et al. 2000) with emission measures of $\sim 4 \times 10^{61} \mathrm{~cm}^{-3}$ and $\sim 10^{61} \mathrm{~cm}^{-3}$, respectively. RX J0059.2-7138 was observed at a luminosity of $2.5 \times$ $10^{38} \mathrm{erg} \mathrm{s}^{-1}$, i.e. both luminosity and emission measure were a factor of 40 higher compared to the maximum values observed from RXJ0103.6-7201. On the other hand, for EXO 053109-6609.2 an intrinsic source luminosity of $4.6 \times 10^{37} \mathrm{erg} \mathrm{s}^{-1}$ was reported which yields a ratio $E M\left[\mathrm{~cm}^{-3}\right] / L_{\mathrm{x}}\left[\mathrm{erg} \mathrm{s}^{-1}\right]$ of $8.7 \times 10^{23}$ a factor of 20 higher than for RX J0103.6-7201. Sasaki et al. (2003) report two more SMC pulsars which exhibit a soft emission component. We re-analyzed the EPIC-pn spectra of RXJ0101.3-7211 and AXJ0103-722 in a consistent way as was done for RX J0103.6-7201. Keeping the temperature of the thermal plasma component fixed at $0.15 \mathrm{keV}$ and using a common $N_{\mathrm{H}}$ for soft and hard (power-law) component we derive $E M$ and intrinsic source luminosity $(0.2-10.0 \mathrm{keV})$. The results, together with the values found in the literature, are included in the bottom panel of Fig. 6. They are consistent with the linear relation between $E M$ (or luminosity of the soft component) and total source luminosity derived for RX J0103.6-7201 with $E M\left[\mathrm{~cm}^{-3}\right]=4 \times 10^{22} \times L_{\mathrm{x}}\left[\mathrm{erg} \mathrm{s}^{-1}\right]$ (plotted as straight line). 
Table 2. Optical identifications.

\begin{tabular}{lcccccc}
\hline \hline \multicolumn{1}{c}{ Colours (catalogue) } & RA and Dec. (2000.0) & $V \operatorname{mag}$ & $B-V$ & $U-B$ & $V-R$ & $V-I$ \\
\hline$U B V R$ (Massey 2002) & $01^{\mathrm{h}} 03^{\mathrm{m}} 37^{\mathrm{s}} 46-72^{\circ} 01^{\prime} 33^{\prime \prime} .2$ & 14.54 & -0.06 & -0.99 & -0.08 & - \\
$U B V I$ (Zaritsky et al. 2002) & $01^{\mathrm{h}} 03^{\mathrm{m}} 37.52-72^{\circ} 01^{\prime} 33^{\prime \prime} .0$ & 14.65 & -0.11 & -1.08 & - & -0.04 \\
\hline
\end{tabular}

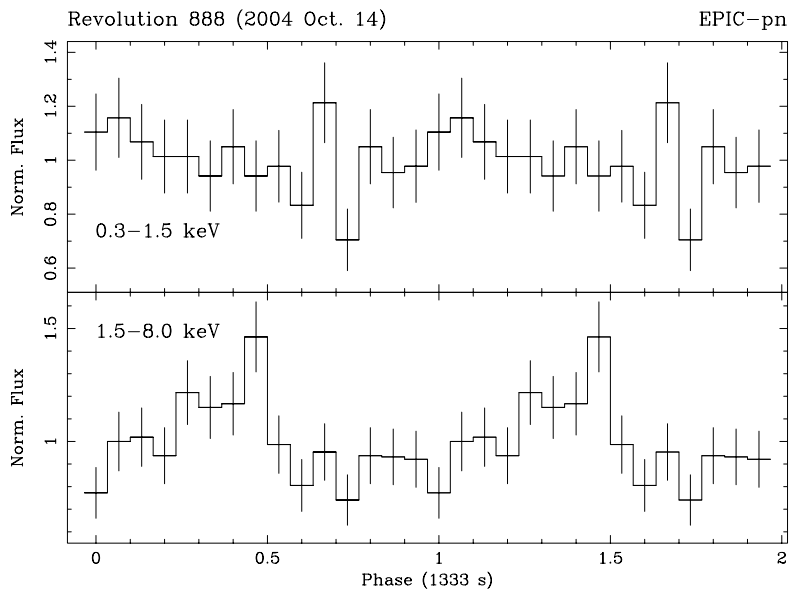

Fig. 4. EPIC-pn light-curve from revolution 888 in soft and hard energy bands folded with the pulse period.

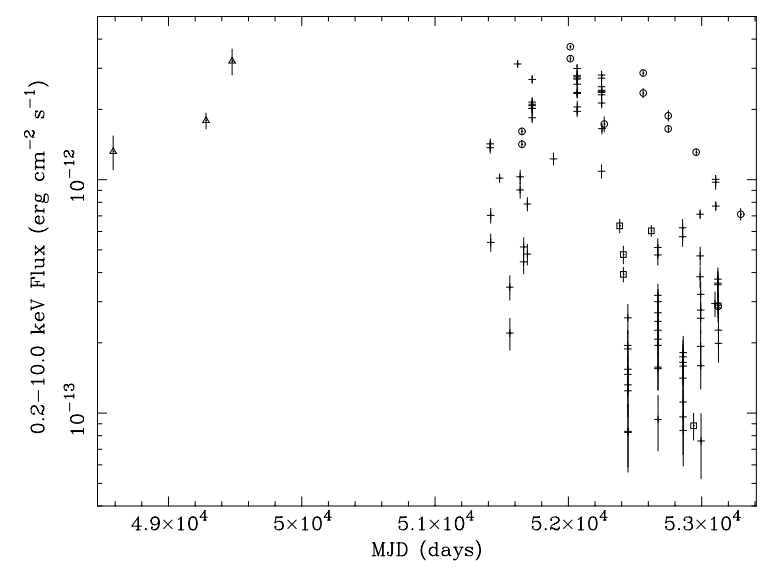

Fig. 5. X-ray flux variations from RXJ0103.6-7201 since the first ROSAT (triangles) observation in Nov./Dec. 1991. Circles mark XMM-Newton observations with fluxes directly derived from spectral fits to the EPIC-pn spectra (which yield two data points when two read-out modes were used. All other fluxes were converted from count rates assuming a power-law + MEKAL model with typical parameters as derived from the EPIC spectra (see text). Crosses indicate Chandra observations and squares XMM-Newton observation with the source at low intensity.

Here we use $E M$ instead of luminosity of the soft component to be able to compare with published data.

Hickox et al. (2004) explored four possible mechanisms for the origin of the soft excess in X-ray pulsars: 1) re-processing of hard X-rays by optically thick, dense material near the neutron star; 2) re-processing by optically thin material; 3) thermal emission from collisionally ionized plasma; and 4) soft emission from the accretion column and the neutron star surface. They conclude that for luminous sources $\left(L_{\mathrm{x}}>10^{38} \mathrm{erg} \mathrm{s}^{-1}\right)$ only case 1) can explain the strong soft component while
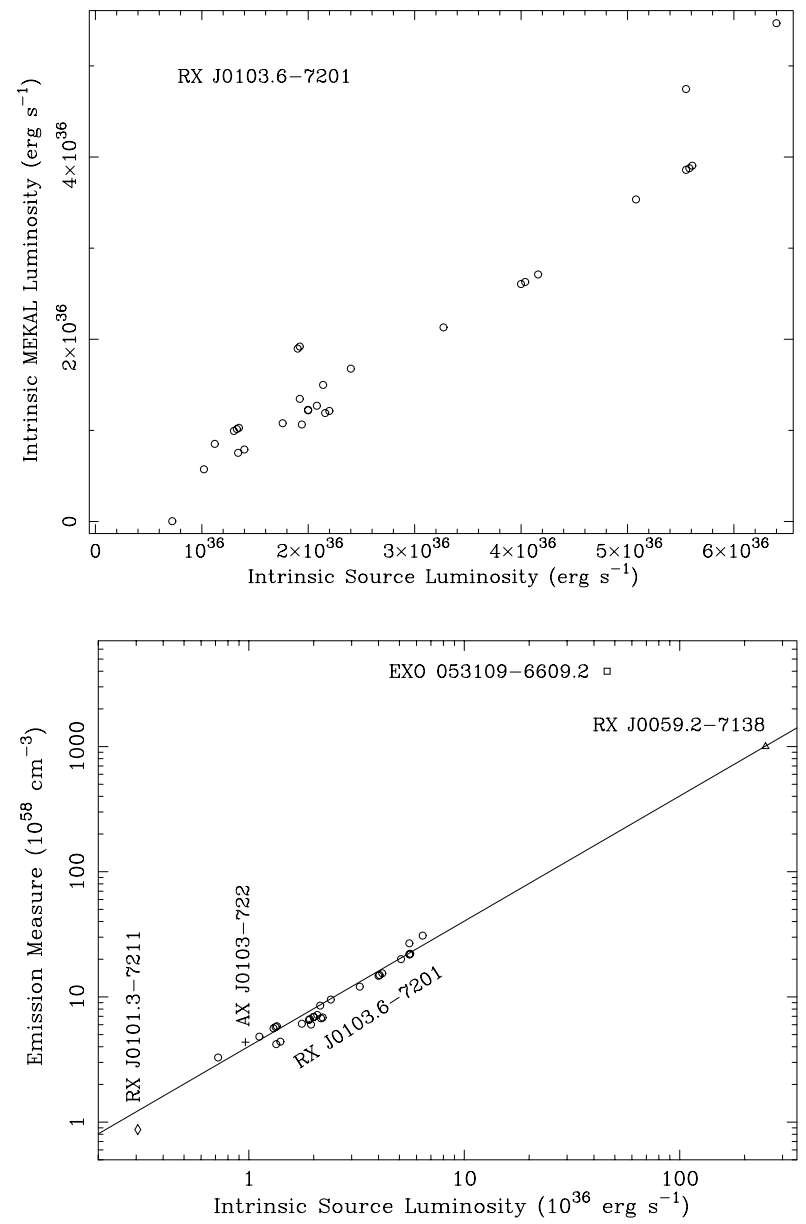

Fig. 6. Top: luminosity of the soft spectral component (modeled as MEKAL component) in the EPIC spectra of RX J0103.6-7201 vs. total source luminosity. Both luminosities are source intrinsic, i.e. corrected for photo-electric absorption. Bottom: emission measure of the thermal plasma component as function of the total source intrinsic luminosity including results from other Be/X-ray binaries.

for luminosities below $10^{36} \mathrm{erg} \mathrm{s}^{-1}$ all three other cases can contribute.

The tight correlation between the luminosity of the soft spectral component and the total source luminosity we found for RX J0103.6-7201 over a factor of 10 in luminosity and moreover extending to three orders of magnitude when including results from three other SMC Be/X-ray binary pulsars strongly suggests that there is a single mechanism at work over a wide range of source intensity. The correlation as such makes emission from a collisionally ionized plasma unlikely and supports the models involving re-processing of the X-ray emission from the neutron star. Therefore, the MEKAL model is actually not the proper model to derive parameters of the emitting plasma like temperatures or elemental abundances. However, it 
best fits the soft part of the EPIC spectra among all available simple XSPEC models and allows us to accurately determine fluxes.

The pulsed fraction observed from RXJ0103.6-7201 is largely reduced (if present at all) at energies below $1.5 \mathrm{keV}$. Similarly, no pulsations at energies below $0.4 \mathrm{keV}$ were seen from the $2.76 \mathrm{~s}$ pulsar RX J0059.2-7138 (Kohno et al. 2000) which indicates a size for a re-processing region with $r>$ $c \times P_{\text {spin }} \sim 10^{11} \mathrm{~cm}$. To smear out longer pulse periods the size must be correspondingly larger and for RX J0103.6-7201 would require a size $>4 \times 10^{13} \mathrm{~cm}$. An independent very rough estimate for the size of the re-processing region can be derived from the emission measure $\int n_{\mathrm{H}} n_{\mathrm{e}} \mathrm{d} V \sim 4 \pi / 3 r^{3} \times n_{\mathrm{H}}^{2}$ and the absorbing column density $\int n_{\mathrm{H}} \mathrm{d} r \sim r \times N_{\mathrm{H}}$. For $E M$ in the range of $(3-30) \times 10^{58} \mathrm{~cm}^{-3}$ and a typical local $N_{\mathrm{H}}$ of $6 \times$ $10^{21} \mathrm{~cm}^{-2}, \mathrm{r}$ is in the range $2 \times 10^{14} \mathrm{~cm}$ to $2 \times 10^{15} \mathrm{~cm}$ (increasing with higher $E M$ and hence source luminosity). If the large absorption observed from revolution 888 for the powerlaw component is caused by dense stellar matter close to the Be star, than these latter size estimates must be regarded as upper limit. A lower limit of $1 \times 10^{12} \mathrm{~cm}$ can then be derived using the high $N_{\mathrm{H}}$ value. These simple estimates argue for reprocessing of the hard X-rays in a large part of the optically thin stellar wind. The correlation of soft flux with source intensity for four Be/X-ray binaries in the SMC suggests that this mechanism is the dominant process to create the soft spectral component at least in HMXBs with a Be star as mass donor.

It remains to be explained why EXO 053109-6609.2 exhibits a much stronger soft component compared to the SMC $\mathrm{Be} / \mathrm{X}$-ray pulsars. It is unlikely that the higher metallicity of the LMC can cause a factor of 20 increase in reprocessing efficiency. EXO 053109-6609.2 has shown a more complex spectrum during the XMM-Newton observation reported by Haberl et al. (2003) which may be interpreted as partial covering of the X-ray source. Hence, a large fraction of the hard emission might be completly blocked and the total source luminosity underestimated. Other $\mathrm{Be} / \mathrm{X}$-ray binaries in the LMC with a soft emission component need to be observed to verify whether
EXO 053109-6609.2 is an exceptional case (e.g. A 0538-66, Mavromatakis \& Haberl 1993).

Acknowledgements. We are grateful to Manami Sasaki for providing the EPIC spectra of SMC pulsars with soft emission for our re-analysis. The XMM-Newton project is supported by the Bundesministerium für Bildung und Forschung / Deutsches Zentrum für Luft- und Raumfahrt (BMBF / DLR), the Max-Planck-Gesellschaft and the Heidenhain-Stiftung.

\section{References}

Coe, M. J., Edge, W. R. T., Galache, J. L., \& McBride, V. A. 2005, MNRAS, 356, 502

Cutri, R. M., Skrutskie, M. F., van Dyk, S., et al. 2003, VizieR Online Data Catalog, 2246, 0

Haberl, F., Angelini, L., \& Motch, C. 1998, A\&A, 335, 587

Haberl, F., Dennerl, K., \& Pietsch, W. 2003, A\&A, 406, 471

Haberl, F., Filipović, M. D., Pietsch, W., \& Kahabka, P. 2000, A\&AS, 142,41

Haberl, F., \& Pietsch, W. 2004, A\&A, 414, 667

Haberl, F., \& Sasaki, M. 2000, A\&A, 359, 573

Haberl, F., White, N. E., \& Kallman, T. R. 1989, ApJ, 343, 409

Hickox, R. C., Narayan, R., \& Kallman, T. R. 2004, ApJ, 614, 881

Kohno, M., Yokogawa, J., \& Koyama, K. 2000, PASJ, 52, 299

Majid, W. A., Lamb, R. C., \& Macomb, D. J. 2004, ApJ, 609, 133

Massey, P. 2002, ApJS, 141, 81

Mavromatakis, F., \& Haberl, F. 1993, A\&A, 274, 304

Mewe, R., Gronenschild, E. H. B. M., \& van den Oord, G. H. J. 1985, A\&AS, 62, 197

Meyssonnier, N., \& Azzopardi, M. 1993, A\&AS, 102, 451

Sako, M., Liedahl, D. A., Kahn, S. M., \& Paerels, F. 1999, ApJ, 525, 921

Sasaki, M., Haberl, F., \& Pietsch, W. 2000, A\&AS, 147, 75

Sasaki, M., Pietsch, W., \& Haberl, F. 2003, A\&A, 403, 901

Strüder, L., Briel, U., Dennerl, K., et al. 2001, A\&A, 365, L18

Turner, M. J. L., Abbey, A., Arnaud, M., et al. 2001, A\&A, 365, L27

Yokogawa, J., Imanishi, K., Tsujimoto, M., Koyama, K., \& Nishiuchi, M. 2003, PASJ, 55, 161

Zaritsky, D., Harris, J., Thompson, I. B., Grebel, E. K., \& Massey, P. 2002, AJ, 123, 855 